\title{
Crystal Growth and Solute Trapping
}

\section{Citation}

Aziz, Michael J. Crystal Growth and Solute Trapping. 1984. Materials Research Society Symposium Proceedings 23: 369-374.

\section{Published Version}

http://www.mrs.org/s_mrs/sec.asp?CID=1727\&DID=38980\&SID=1

\section{Permanent link}

http://nrs.harvard.edu/urn-3:HUL.InstRepos:3306021

\section{Terms of Use}

This article was downloaded from Harvard University's DASH repository, and is made available under the terms and conditions applicable to Other Posted Material, as set forth at http:// nrs.harvard.edu/urn-3:HUL.InstRepos:dash.current.terms-of-use\#LAA

\section{Share Your Story}

The Harvard community has made this article openly available.

Please share how this access benefits you. Submit a story.

Accessibility 
CRYSTAL GROWTH AND SOLUTE TRAPPING

MICHAEL J. AZIZ

Division of Applied Sciences, Harvard University, Cambridge, MA 02138 
CRYSTAL GROWTH AND SOLUTE TRAPPING

MICHAEL J. AZIZ*

Division of Applied Sciences, Harvard University, Cambridge, MA 02138

\section{ABSTRACT}

A simple model for solute trapping during rapid solidification is presented in terms of a single unknown parameter, the interfacial diffusivity $D_{i}$. A transition from equilibrium segregation to complete solute trapping occurs over roughly an order of magnitude in growth speed, as the interface speed surpasses the maximum speed with which solute atoms can diffuse across the interface to remain ahead of the growing crystal. This diffusive speed is given by $D_{1} / \lambda$, where $\lambda$ is the interatomic spacing, and is typically of the order 10 meters per second. Comparison is made with experiment. The steady-state speed of a planar interface is predicted by calculating the free energy dissipated by irreversible processes at the interface and equating it to the available driving free energy. A solute arag term and an intrinsic interfacial mobility term are included in the dissipation calculations. Steady-state solutions are presented for Bi-doped Si during pulsed laser annealing.

\section{INTRODUCTION}

Laser annealing expeximents have reached a crystal growth regime where deviations from local equilibrium are obvious and interface motion is no longer heat-flow limited. These experiments allow us the opportunity to study the interface kinetics in high-mobility systems for the first time. In this paper I describe a simple model for the kinetics of the fundamental atomic processes occurring at the interface during rapid solidification of binaxy alloys, one of which, of course, is doped silicon. The result is a pair of "interface response functions" which predict (a) how much impurity should be incorporated into the solid, and (b) how fast the interface should move; in terms of the local conditions at the interface, namely temperature and composition.

The experiments $[1,2]$ that have inspired the modeling efforts have shown that the chemical potential of the minor component of a binary alloy often increases as the partition coefficient $k$ approaches unity during rapid solidification. A number of plausible models have been proposed [3-9] to explain how the host atoms persuade the impurity atoms to increase their chemical potential and join the crystal. The basis for the model described in this paper is shown in Fig. 1, which illustrates the fundamental difference between crystallization and interdiffusion according to the model for collision-limited growth of pure systems with simple intexatomic potentials, which has been developed by Turnbull and coworkers [10]. A crystallization event consists of an atom shifting its position a small distance to move from a potential well in the liquid structure to a well on the crystal lattice as the solid arrows indicate. Since no bonds must be broken, the activation barrier for this reaction ought to be quite small; in the Turnbull collision-limited growth model the barrier is zero. A diffusive jump, on the other hand, is a different atomic process in this system. Position shifts of a fraction of an interatomic distance do not accomplish interdiffusion, which requires a good deal of cooperation from neighboring atoms. Since the crystallization process can occur without 
these difficulties, the growth speed $u$ can very much exceed the maximum diffusive speed given by $u_{D}=D_{i} / \lambda$, where $D_{i}$ is the coefficient for interdiffusion across the interface and $\lambda$ is the interatomic distance. The model has received recent support from the molecular dynamics calculations of Broughton et al. [11], as disussed by Jackson in this session [12].

Fig. 1. Choreography of Solute Trapping. In order to avoid incorporation into the crystal (solid arrow), the "B" atom must:

i) ESCAPE from its potential well (dashed arrow) as the well gains energy and moves to the lattice site (solid arrow),

ii) PUSH " $\mathrm{A}$ " atoms out of the way,

iii) BE PEPLACED with an " $A$ " atom on the lattice site.

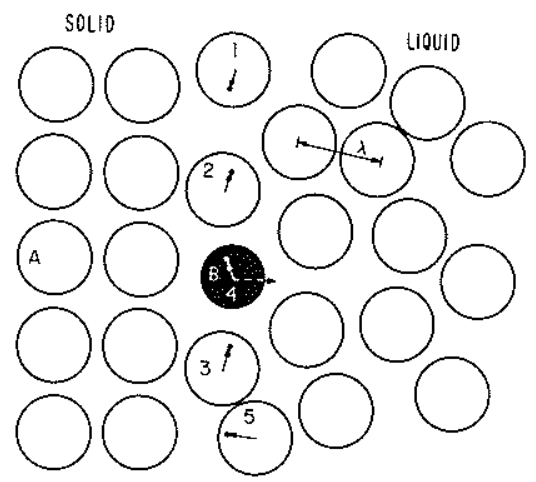

Fig. 2. Preliminary comparison with experiment. Variation of segregation coefficient $x$ with velocity. Theory for dilute solution limit. Experimental velocities unom calculated assuming $\mathrm{T}_{i}=\mathrm{T}_{M}$.

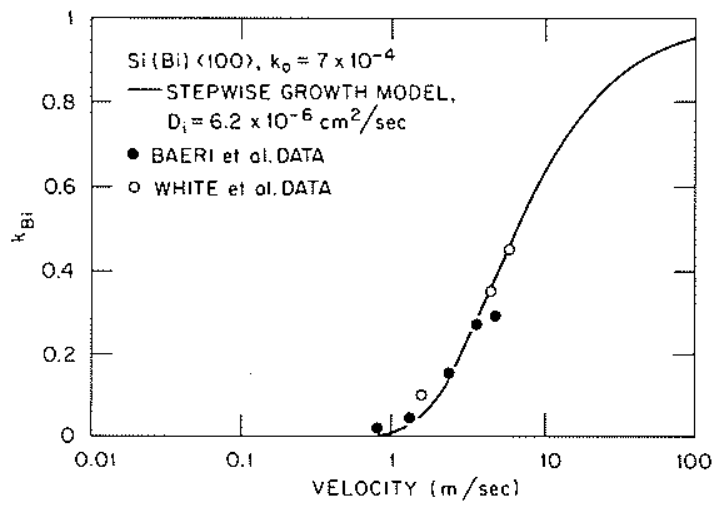

SOLUTE TRAPPING MODEL

The solute trapping model described herein considers solute-solvent redistribution, which is necessary to avoid solute incorporation into the crystal, to be the same as the interdiffusive process described above. Thus a solute atom must accomplish three things to avoid crystalization: It must escape from its potential well, as shown by the dashed arrow in Fig. 1, as the energy of the well increases and the well moves to the lattice site. It must push solvent atoms out of the way. And it must be replaced by a solvent atom on the lattice site, or else it will be pushed right back to where it came from. This process occurs in the interface region as the atoms attempt to establish equilibrium compositions on either side of the moving interface. As the interface passes, solute-solvent 
redistribution can only occur for a limited time. Before the interface arrives, the driving force for redistribution is zero; afterwards, atomic mobilities are too low to permit significant interdiffusion. When $u<u^{\prime}$ there is plenty of time for the atoms at the interface to equilibrate. $B^{\prime}$ the growth velocity becomes greater, solute and solvent atoms have less time while they are in the interface region to interdiffuse and maintain local equilibrium. When $u \gg u$, the atoms have no, time to interdiffuse; the interface passes and locks the liquid composition into the bulk of the solid before the atoms have a chance to react.

In the formalism of this model, crystal growth and solute-solvent redistribution are written as separate reactions with different mobilities. solute trapping follows simply and directly as the interface speed surpasses $u_{\mathrm{D}}$. The derivation and discussion of the solute trapping model for dilute and concentrated solutions during stepwise and continuous interface motion can be found elsewhere [13-16]. The results for stepwise growth and for continuous growth at the same interface speed differ quantitatively but exhibit the same qualitative features. A transition from equilibrium segregation $\left(k=k_{0}\right)$ to complete solute trapping $(k=1)$ is centered at the diffusive speed, as shown by the curve in Fig. 2 .

Plotted along with the curve are the solute trapping data of Baeri et al. [17] and of White et al. [18] for $S i(B i)\langle 100\rangle$. The single unknown parameter in the model is the interface diffusivity $D_{i}$, which has been chosen to fit the data. When plotted on a logarithmic velocity scale like this, the only effect of varying $D_{i}$ is a shift of the curve to the left or right. Thus the slope of the curve is independent of $D_{i}$, and when it matches that of the data it is an encouraging sign. The comparison is only preliminary because the experimental velocities are not measured. Nominal velocities $u_{\text {nom }}$ have been calculated from complicated numerical solutions of the heat equation for pure silicon using the heat-flow-limit assumption that the interface temperature $T_{i} i s$ at the metting point of pure silicon,

$T_{M}$. The possible errors introduced by such assumptions are discussed later. The curious phenomenon called "saturation" is seen in the data of Baeri et al. but apparently not in those of white et al. Most simple solute trapping models yield smooth, fairly straight curves when plotted as linear $k$ vs. $\log u$, and "saturation", which Baeri has also seen in Si(In) [19], may be pointing out inadequacies in the simple models if the velocity calculations are correct. One possible way to account for "saturation" is to assign to the interface thermodynamic properties different from those of either bulk phase $[3,4,6,8,20]$. Further experimental investigation of this effect is needed if we are to understand thoroughIy the kinetics of the solute trapping process.

\section{DISSIPATION THEORY}

Solute trapping models require knowledge of the intexface velocity. Deviations from heat-flow-limited growth are expected at high speeds and solute concentrations. A dissipation-theory treatment $[15,16]$ can be used to predict the steady-state velocity of the interface as a function of its temperature and composition. If the sum of the physical forces on a unit area of the interface is zero, its velocity will remain unchanged. From the driving force for solidification we subtract the "solute drag" force due to the snowplow-like action of the interface upon the solute, and the "interface friction" force due to the limited mobility of the interface in the pure system. Alternatively and equivalently, we are requiring that the available driving free energy be dissipated by the irreversible reactions occurring at the interface. In the steady state, the driving free energy does no work on the surroundings and so it must be entirely dissipated by irreversible processes during the transformation. If we can calculate the dissipation and the driving free energy as functions of velocity, we have an equation that predicts the growth velocity. Ignoxing heat conduction, 
the two dissipative processes at the interface are the aforementioned crystallization and redistribution reactions. The dissipative terms associated with them are, respectively, the "interface fxiction enexgy" and the "solute drag energy" $[21,22]$. By assuming interface friction to be the same for alloys as for pure systems, we can calculate these terms $[15,16\}$ and make predictions. The result is that in some regimes the interface velocity can be significantly slower than the reported "isotherm velocities" of heat-flow calculations.

\section{APPLICATION TO LASER ANNEALING}

In order to apply these ideas to laser annealing, some account must be made of the effect of an undercooled interface upon the temperature profile during regrowth. The heat-flow problem in lasex annealing is a complex one. Since finite-element heat-flow programs that account properly for interfacial undercooling are not yet available, a crude analysis of the effect of undercooling [23] is summarized below. Heat-flow calculations [24, 25] have in the past solved the heat equation for temperature profiles during regrowth by assuming the interface temperature $\mathrm{T}_{i}$ to be fixed at the melting point $\mathrm{T}_{M}$. If the heat-flow problem were strictly linear and the details of how the undercooling is set up were unimportant, the actual temperature profile during regrowth would be scaled down relative to the calculated one by the factor $\left(T_{1}-T_{A m b}\right) /\left(T_{M}-T_{A m b}\right)$, where $T_{A m b}$ is the ambient substrate temperature. All gradients would also scale accordingly and therefore the regrowth speed, which is proportional to the gradient in the solid at the interface, would do the same. (The discrepancy between measured and calculated regrowth speeds at the "amorphization threshold" has been interpreted in this manner; the undercooling necessary to account for it is $\sim 230 \mathrm{~K}$. The analysis yields an estimate of the melting point of amorphous silicon of $\mathrm{T}_{a l} \sim 1180^{\circ} \mathrm{C}$ and an intrinsic mobility of the $\langle 100\rangle$ crystal-melt interface of $u_{0} \sim 34 \mathrm{~m} / \mathrm{s}$ [23].)

This heat-flow analysis has been combined with dissipation theory to predict the effect of solute concentration upon the regrowth velocity. Heat-flow calculations predict a nominal regrowth velocity which is independent of solute concentxation. The reduced driving force for the solidification of impure material, the interface friction texm, and the concentration-dependent solute drag term in the dissipation calulations reduce the interface speed relative to the heat-flow-limit predictions. The equations are solved simultaneously along with the crude analysis of the gradients mentioned above.

One result is presented in Fig. 3, which shows the velocity u predicted by dissipation theory in terms of the "strength of quench", or nominal velocity $u_{n o m}$ predicted by the heat flow calculations that assume $T_{i}=T_{M}$. The dissipation calculations are done for bismuth-doped silicon. The required input parameters are the diffusive speed $u_{D^{\prime}}$ taken from a fit to the $k_{\mathrm{Bi}}(u)$ data; the intrinsic interface mobility $u_{0}$, taken from [23]; and the thermodynamics of supersaturated $S i(B i)$, for which very crude guesses were made. A parabolic regular solution was assumed for the solid and the liquid with interaction parameters of 23 and $10 \mathrm{kcal} / \mathrm{mole}$, respectively. The melting point of pure diamond cubic $\mathrm{Bi}$ was taken to be $100 \mathrm{~K}$. Due to the poor thermodynamic model, the crude treatment of the heat flow, and the steady-state nature of the analysis, the results can be thought of as only qualitative. But the trends will not change. For sufficiently dilute solutions (i.e. curve labelled "- " in Fig. 3), the effects are negligible below about $10 \mathrm{~m} / \mathrm{s}$. But for a given quenching condition, the velocity drops as the $\mathrm{Bi}$ dose increases. The magnitude of the effect is considerable at velocities where the partition coefficient is far from both $k_{0}$ and 1. The solute drag force turns out to be greatest in this regime [15]. For large enough solute concentrations, three solutions are found for some quenching 
Fig. 3. Predicted variation of actual velocity with nominal heat-flowlimit velocity in Si(Bi), for various mole fractions $x_{L}$ of solute in the bulk of the liquid phase.

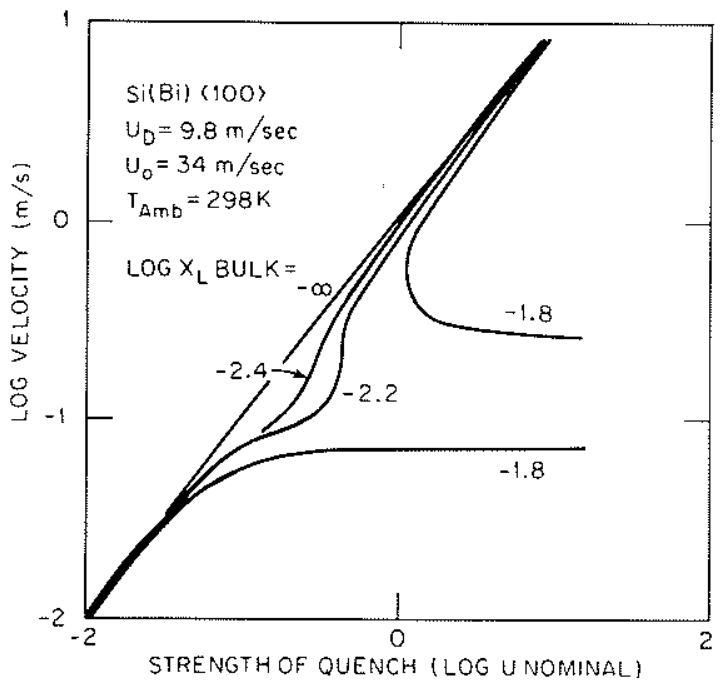

conditions, as in the curves labelled "-1.8" in Fig. 3. The lower branch corresponds to a slow-moving interface laden with solute. The midale branch is probably unstable. And the top branch corresponds to an interface which has "broken away" from its solute atmosphere. The analogy with the treatment of grain boundary migration [21] is evident. However, morphological instability during solidification has not been taken into account; cellular breakdown may make most of the lower branch unobservable.

Comparison with experiment. Galvin and coworkers have been looking for this effect; they appear to have found it [26]. For identical laser annealing conditions, indium-doped $\mathrm{Si}$ grows back slower than pure Si. In the foreseeable future quantitative comparisons of theory and experiment should be possible.

\section{CONCLUSIONS}

A simple solute trapping model can be understood as a direct consequence of the collision-limited growth model discussed by Turnbull and by Jackson, which asserts that crystal growth can occur much more rapidly than interdiffusion. The interface velocity can be predicted by balancing the physical forces on the interface. The theory predicts that the presence of dopants reduces the regrowth speed from laser-induced melting. There are preliminary indications of experimental confirmation of the predictions.

One of the major advantages of this model is that the calculations are simple enough to be performed by anyone. The trouble with a model this simple is that it must gloss over a lot of complexities and it certainly cannot be correct to several significant digits. However, with only a single free parameter it seems to predict the right trends. 
Spaepen for much helpful discussion; and to D. Turnbull for guidance. This work was supported in part by an Allied Corp. Fellowship Grant and by National Science Foundation Grant DMR-79-23597.

\section{REFERENCES}

*Present address: Solid State Division, Oak Ridge National Laboratory, Oak Ridge, TN 37831 .

[1] J.C. Baker and J.W. Cahn, in Solidification (ASM, Metals Park, Ohio, 1970).

[2] C.W. White, S.R. Wilson, B.R. Appleton, and F.W. Young Jr., J. Appl. Phys. 51, 738 (1980).

[3] J.C. Bxice, The Growth of crystals From the Melt (North-Holland, Amsterdam, 1965), pp. 63-7.

[4] R.N. Hall, J. Phys. Chem. 57, 836 (1953).

[5] M. Hillert and B. Sundman, Acta Metal1. 25, 11 (1977).

[6] J.C. Baker, reported by J.W. Cahn, S.R. Coriell and W.J. Boettinger, in Laser and Electron Beam Processing of Materials, ed. C.W. White and P.S. Peercy (Academic, New York, 1980), pp. 89-103.

[7] K.A. Jackson, G.H. Gilmex and H.J. Leamy, pp. 104-110 of [6].

[8] G.H. Gilmer, Mat. Res. Soc. Symp. Proc. 13, 249 (1983).

[9] R.F. Wood, Appl. Phys. Lett. $37,302(19 \overline{80})$.

[10] S.R. Coriell and D. Turnbull, Acta Metall. 30, 2135 (1982). See also references in $\{15\}$.

[11] J.Q. Broughton, G.H. Gilmer, and K.A. Jackson, Phys. Rev. Lett. 49 , 1496 (1982).

[12] K.A. Jackson, these proceedings.

[13] M.J. Aziz, "Kinetics of Crystallization of $\mathrm{B}_{2} \mathrm{O}_{3}$ Under Pressure and Theory of Motion of the crystal-Melt Intexface at Wide Departures from Equilibrium", Ph.D. thesis, Harvard University (University Microfilms International, Ann Arbor, Michigan, 1984).

[14] M.J. Aziz, J. Appl. Phys. 53, 1158 (1982).

[15] M.J. Aziz, Appl. Phys. Lett. 43, 552 (1983).

[16] M.J. Aziz, in Rapid Solidification processing Principles and Technologies III, ed. R. Mehrabian (Nat. Bur. Standards, Gaithersburg MD, 1982), pp. 113-7.

[17] P. Baeri, G. Eoti, J.M. Poate, S.U. Campisano, and A.G. Cullis, Appl. Phys. Lett. 38, 800 (1981).

[18] C.W. White, B.R. Appleton, B. Stritzker, D.M. Zehner, and S.R. Wilson, in Mat. Res. Soc. Symp. Proc. 1, 59 (1981).

[19] P. Baeri, reported by C.W. White, D.M. Zehner, S.U. Campisano and A.G. Cullis, in Surface Modification and Alloying By Laser, Ion, and Electron Beams, ed. J.M. Poate, G. Foti and D.C. Jacobson (Plenum, New York, 1983), pp. 93-6.

[20] Ref. [13], pp. 76-82.

[21] J.W. Cahn, Acta Metall, 10,789 (1962).

[22] M. Hillert and B. Sundman, Acta Metall. 24, 731 (1976).

[23] Ref. [13], chapter 5.

[24] R.F. Wood and G.E. Giles, Phys. Rev. B 22, 2923 (1981).

[25] P. Baeri, S.U. Campisano, G. Foti and E. Rimini, J. Appl. Phys. 50 $788(1979)$.

[26] G.J. Galvin, J.W. Mayer and P.S. Peercy, these proceedings. 